\title{
The Effect of Elementary School Soccer Instruction Using Virtual Reality Technologies on Students' Attitudes toward Physical Education and Flow in Class
}

\author{
Hyun Suk Lee ${ }^{1}$ (D) and Junga Lee ${ }^{2, *(\mathbb{D})}$ \\ 1 Graduate School of Education, Chung Ang University, Seoul 06974, Korea; hslee@cau.ac.kr \\ 2 Faculty of Sports Medicine and Science, Kyung Hee University, Yongin-si, Gyeonggi-do 17104, Korea \\ * Correspondence: jalee@khu.ac.kr; Tel.: +82-(31)-201-2738
}

Citation: Lee, H.S.; Lee, J. The Effect of Elementary School Soccer

Instruction Using Virtual Reality

Technologies on Students' Attitudes toward Physical Education and Flow in Class. Sustainability 2021, 13, 3240. https://doi.org/10.3390/su13063240

Academic Editor:

Emilio Villa-González

Received: 4 February 2021

Accepted: 12 March 2021

Published: 15 March 2021

Publisher's Note: MDPI stays neutral with regard to jurisdictional claims in published maps and institutional affiliations.

\begin{abstract}
We analyzed the effects of an elementary school soccer class using virtual reality technology on students' attitudes toward physical education class and the influence on class flow. The data from 113 elementary school students from Seoul and Gyeonggi-do were analyzed. Students were divided into groups that received either virtual reality or traditional classes (e.g., playing on a playground). Data were analyzed with three-way analyses of variance. Results revealed that students who participated in the virtual reality classes had more confidence, concentration, and experienced more flow (specifically, attention to PE class, integration of ability, challenge, and sense of control) than students who participated in the traditional class. This suggests that virtual reality technology positively affected students' attitudes and flow. In subsequent research, it is necessary to develop and disseminate a variety of virtual reality sports programs and to conduct studies with students from various grade levels.
\end{abstract}

Keywords: virtual reality; attitude toward class; physical education; flow

\section{Introduction}

Faced with the rapidly changing era of the Fourth Industrial Revolution, several efforts have been made to prepare for changes in various parts of the world, and the field of physical education (PE) has also undergone changes [1]. Specifically, physical educators must develop and use technology effectively when educating students [2]. However, since most PE teachers-from elementary school to university - tend to mainly demonstrate and practice in front of students, most students participate in PE classes passively [3]. Additionally, environmental problems such as a lack of a budget for playgrounds and indoor gymnasium facilities are often mentioned in most Korean schools [4].

Moreover, in outdoor PE activities, not only facilities but also weather and fine dust are key obstacles. Recently, rain, drought, heatwaves, and cold weather have negatively affected PE classes [5]. To solve these problems, many educational experts have offered various alternatives in PE, including technological innovations [6].

Research using VR [7-10] is actively underway in various sports such as dance and ballet. These studies show that the use of VR is more effective than other technological attempts in terms of providing a level of guidance, mode of communication, and type of feedback to users. Therefore, it is necessary to study how VR affects students in school PE that are taught through sports.

Little research has been conducted on PE class using VR. A study on the current status and development plan of an ICT-convergence PE class using a VR sports room by Park and his colleagues [11] showed that classes using VR can provide an educational environment suitable for digital native students and solve the problem of the lack of sports facilities. Chang and his colleagues examined the use of VR in PE, and they mentioned that the use of VR is advantageous in that the learner receives customized education. Additionally, 
because VR is a type of media that can produce three-dimensional experiences, it is a learning tool that is more realistic than other multimedia materials and is quite effective in improving achievement [12]. According to Tang and Tsai, the use of VR technology can motivate students and give them a realistic experience [13]. Additionally, VR allows students to proactively obtain information by selecting the image they want rather than passively receiving it [14]. This allows learners and teachers to interact with each other beyond the limits of geographical space [15]. Furthermore, there is an ongoing debate that is focused on the potential of VR that could even extend its possibilities to the exploration of actual physical space, such as integrating multimedia into VR or using perspectives that could not be reached in the real world $[16,17]$. As such, VR will be a major factor in the change of the educational ecosystem and it is highly likely that it will affect future education $[13,15]$. In other words, VR is a model of future education that more effectively supports experiential learning [14,18].

According to the Korean Ministry of Culture, Sports and Tourism, from March 2018, elementary school students can enjoy school sports safely by the dissemination of VR sports rooms in 178 elementary schools nationwide [19]. The VR sports room is a fusion of information and communication technology (ICT) and PE; however, it is also possible to implement the technology with other subjects, thus highlighting the potential for future development. Additionally, VR can be used to solve the aforementioned problems of lack of exercise space, fine dust, and weather.

Research on PE classes using technology has recently started in Korea. Research on PE classes using ICT, social networking services, and mobile applications is underway. However, it has been difficult to find research related to PE classes and the use of VR, augmented reality, big data, and artificial intelligence. Therefore, verification of its educational effectiveness should be given priority for VR technology to be used more effectively in PE [11].

Previous studies [6,20-23] related to ICT-based PE classes have been conducted on blended learning, smart learning, flipped learning, and web-based research; however, there have been no studies on the effects of PE classes using VR technology on students' attitude toward PE and the class flow. According to a press release by the Korean Ministry of Culture, Sports and Tourism [24] on participation in sports by teenagers, soccer has the highest participation rate $(24.3 \%)$ among a variety of sporting activities such as swimming, bodybuilding, yoga, and basketball. Therefore, the purpose of this study was to investigate how an elementary school soccer class using VR technology affects students' attitudes toward PE and the flow in an elementary school PE class. This study aims to inform the applicability of VR in elementary school PE.

\section{Research Hypotheses}

(1) Students participating in soccer classes using VR technology and traditional soccer classes will have differing interests.

(2) Students participating in soccer classes using VR technology and traditional soccer classes will have differing confidence and concentration levels.

(3) Students participating in soccer classes using VR technology and traditional soccer classes will have differing consistency between behavior in class and consciousness, selfdirected experience.

(4) Students participating in soccer classes using VR technology and traditional soccer classes will have differing attention levels to PE classes.

(5) Students participating in soccer classes using VR technology and traditional soccer classes will have differing levels of integration in PE classes.

(6) Students participating in soccer classes using VR technology and traditional soccer classes will have differing levels of challenge and sense of control. 


\section{Materials and Methods}

\subsection{Research Site}

The experimental group, "A" elementary school, set up a VR sports room in January 2020 and has used it in PE classes since March. The control group, "B" elementary school, mainly uses the playground in PE classes and the gymnasium on the days when the weather or fine dust is an issue.

\subsection{Components and Specifications of the VR Sports Room}

The components and specifications of the VR sports room are shown in Figure 1 and Table 1.

Table 1. Specifications of the virtual reality sports room.

\begin{tabular}{|c|c|c|}
\hline Component Name & Specifications & Picture \\
\hline $\begin{array}{l}3 X \\
\text { vision } \\
\text { system }\end{array}$ & $\begin{array}{c}\text { Object recognition method: } \\
\text { - Image processing method using three information } \\
\text { provider cameras } \\
\text { Object recognition accuracy } \\
\text { - Object motion: } 99 \% \text { or more } \\
\text { - Speed: } 95 \% \text { or more } \\
\text { - Position coordinates: } 98 \% \text { or more } \\
\text { Recognition scope (area) } \\
\text { - Adjust according to conditions of actual installation site } \\
\text { - Resolution: } 640 \times 480 \\
\text { - Frame rate: } 180 \text { frames per second } \\
\text { Power supply unit: } 12 \mathrm{~V}, 20 \mathrm{~A}, 240 \mathrm{~W}\end{array}$ & \\
\hline Screen & $\begin{array}{c}\text { Size: } 4100 * 2200 \\
\text { 2. } 3 \text { layers (flame retardant layer, support layer, surface } \\
\text { layer for image reproduction) }\end{array}$ & \\
\hline
\end{tabular}

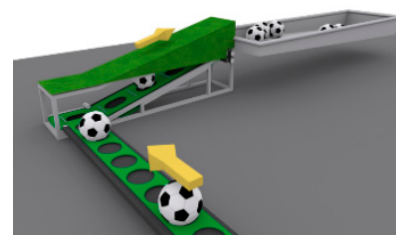

Beam

projector
Rating: AC $220 \mathrm{~V}$

Pixel: $1920 \times 1200$

Brightness: $5000 \mathrm{~lm}$

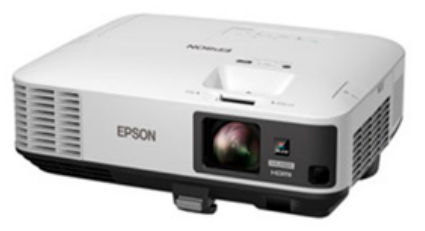




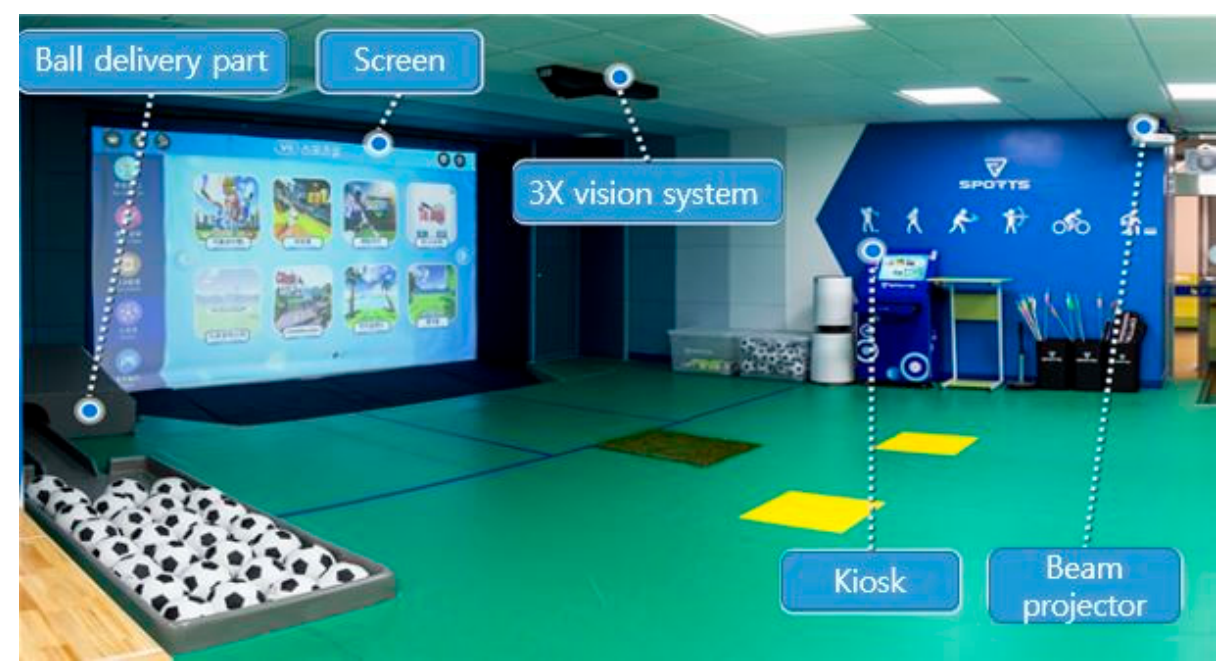

Figure 1. Components of the virtual reality sports room.

The VR program used in this study provided the results of the learner's performance using speech and visual numbers. For example, in practice taking long-distance shots, it informed participants of how many meters they had kicked the ball, and in free kick and penalty kick practice, it informed participants whether they made a goal. Furthermore, the program showed the participants how many points they had made based on the bullseye in the precision shooting practice.

\subsection{Participants}

The population of this study is a grade-five group in elementary school. Using convenience sampling, we selected 120 grade-five students from two elementary schools in Seoul and Gyeonggi-do. The data from 113 respondents, excluding data from seven students who were double-respondents, non-respondents, or absentees, were analyzed.

Researchers were given permission from the principals, PE teachers, and students participating in the research with informed consent. Additionally, the the purpose of the study was fully explained to the participants, the research process, and factors related to safety. Table 2 shows the composition of the study participants.

Table 2. Participant composition.

\begin{tabular}{cccc}
\hline Group & Boys & Girls & Total \\
\hline $\begin{array}{c}\text { Experimental group } \\
\text { ("A" elementary school; } \\
\text { virtual reality group) }\end{array}$ & 25 & 25 & 50 \\
\hline $\begin{array}{c}\text { Control group } \\
\text { (“B” elementary school; }\end{array}$ & 34 & 29 & 63 \\
\hline traditional physical education group) & 59 & 54 & 113 \\
\hline Total & &
\end{tabular}

\section{Research Tools}

\subsection{Questionnaire Composition}

To investigate the effect of VR technology on students' attitudes toward PE and their experience of flow, we used questionnaires by Jang [25] and Hyun [26] and questionnaires by Csikszentmihalyi [27] and Kim [28], respectively. To verify the validity of the questionnaire, it was tested by two professors in the PE field and two PE teachers.

The questionnaire comprised 38 items: 1 concerning personality, 13 concerning attitude, and 24 concerning flow. The latter 37 items were measured with a five-point Likert scale: 1 = "strongly disagree", 2 = "disagree", 3 = "neutral", 4 = "agree", and $5=$ "strongly 
agree". Participants self-reported their responses and participants with missing responses were excluded from analyses.

A reliability test was conducted with 53 students, who were in the same grade as the participants, who completed a preliminary survey. Cronbach's alphas ranged from 768 to 933, indicating the reliability of the questionnaire (see Table 3).

Table 3. Questionnaire composition and reliability.

\begin{tabular}{cccc}
\hline Item & Item Content & Reliability & Question \\
\hline Individuality & Sex & - & 1 \\
\hline Class attitude1 & Interest & 0.924 & 5 \\
\hline Class attitude2 & Confidence & 0.913 & 5 \\
\hline Class attitude3 & Concentration & 0.768 & 3 \\
\hline Flow in class1 & A self-directed experience & 0.933 & 4 \\
\hline Flow in class2 & Attention in physical education class & 0.917 & 5 \\
\hline Flow in class3 & Integration of physical education classes & 0.891 & 5 \\
\hline Flow in class4 & Consensus of class behavior and conscious & 0.890 & 5 \\
\hline Flow in class5 & Challenge and sense of control & 0.896 & 5 \\
\hline & Total & 38 \\
\hline
\end{tabular}

\subsection{Research Design}

The soccer lessons were conducted over five classes. A pre-test was conducted before the first class, and we investigated the effect of the lessons on the experimental and control groups. After that, the experimental group was instructed using the VR technology in the VR sports room and the control group was instructed on the playground. Next, a post-test was conducted on the effects of the PE class on students' attitude and the flow. Finally, data were collected and analyzed using pre- and post-questionnaires (Table 4).

Table 4. Research timetable.

\begin{tabular}{|c|c|c|c|c|c|c|c|}
\hline Time & & & Day 2 & Day 3 & Day 4 & \multicolumn{2}{|c|}{ Day 5} \\
\hline Trials & $\begin{array}{l}\text { Pre- } \\
\text { test }\end{array}$ & $<-$ & & terventi & & $\longrightarrow$ & $\begin{array}{l}\text { Post- } \\
\text { test }\end{array}$ \\
\hline Experimental group & \multirow{2}{*}{$\mathrm{O}_{1}$} & $\mathrm{~T}_{1-1}$ & $\mathrm{~T}_{1-2}$ & $\mathrm{~T}_{1-3}$ & $\mathrm{~T}_{1-4}$ & $\mathrm{~T}_{1-5}$ & \multirow{2}{*}{$\mathrm{O}_{2}$} \\
\hline Control group & & $\mathrm{T}_{2-1}$ & $\mathrm{~T}_{2-2}$ & $\mathrm{~T}_{2-3}$ & $\mathrm{~T}_{2-4}$ & $\mathrm{~T}_{2-5}$ & \\
\hline
\end{tabular}

O post-test (class attitude, flow in class).

\subsection{Class Design}

We planned five soccer classes for fifth graders of both " $\mathrm{A}$ " and " $\mathrm{B}$ " elementary schools. One professor of sport pedagogy, one soccer coach, and two soccer leaders were asked to conduct a content validity test on the contents of the classes. The content of the classes was the same for both groups. The method of teaching (VR or traditional teaching method) according to the place (VR sports room or playground) was applied differently. Furthermore, since teacher factors can affect the results of the study, we created class scenarios for the same topic classes in advance, which was also validated by one professor of sport pedagogy, one soccer coach, and two soccer leaders.

Classes were organized as follows: $10 \mathrm{~min}$ for an introduction and warm-up, $25 \mathrm{~min}$ for lesson development, and $5 \mathrm{~min}$ for discussion and a cool-down to finish the class. Additional details are shown in Table 5 . 
Table 5. Teaching plan for soccer classes.

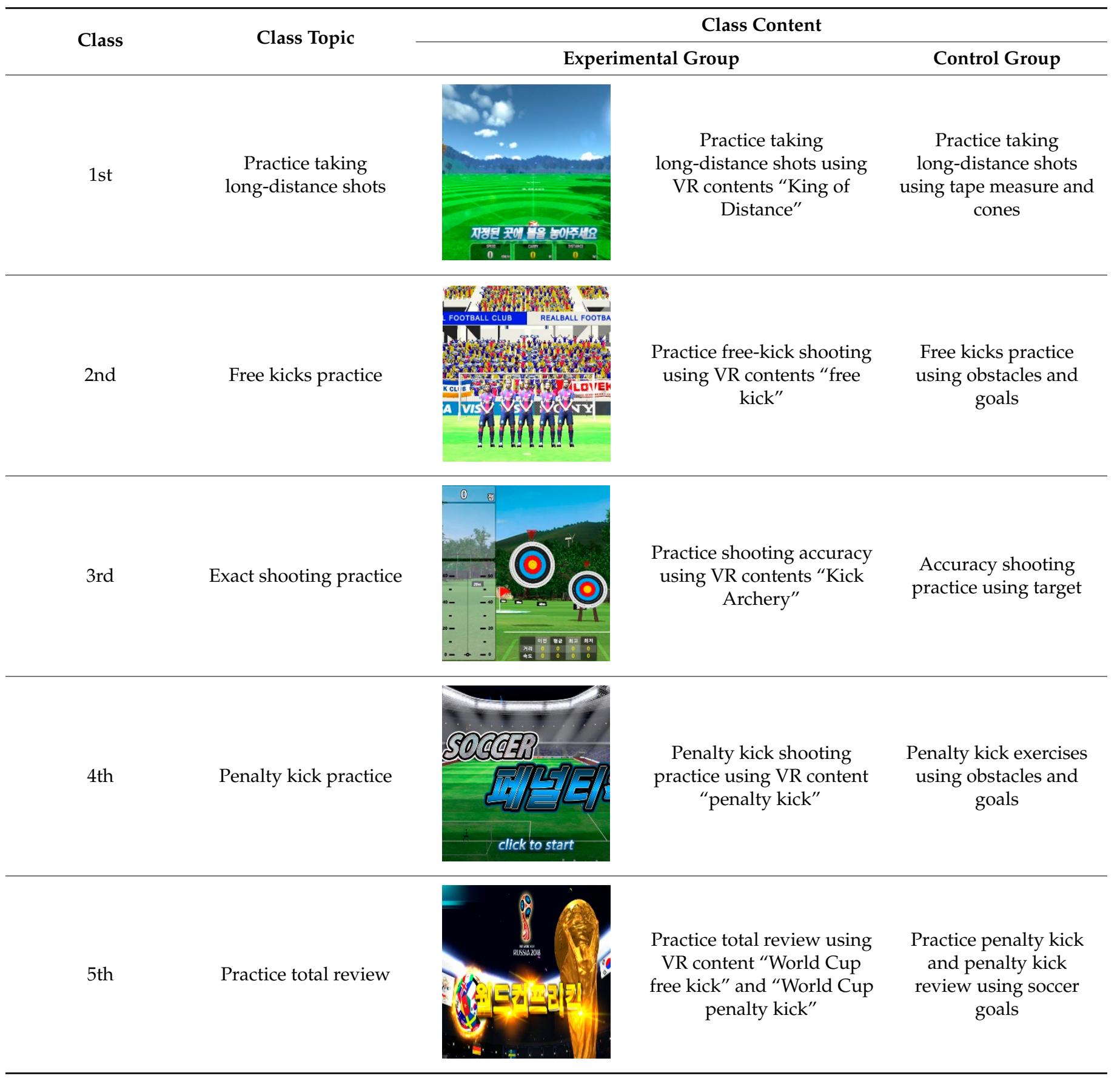

\subsection{Data Analysis}

The collected data were analyzed using SPSS for Windows Version 23.0 (SPSS Inc. 2015) as follows: first, frequency analysis and descriptive analysis were conducted to examine the demographic factors (e.g., participants' sex). Second, Cronbach's $\alpha$ was calculated to determine questionnaire reliability. Third, the independent variables were group (2: VR and traditional instruction), test (2: pre- and post-test), and sex (2: male and female). Dependent variables were class attitude and flow in class. Fourth, a three-way mixed analysis of variance (ANOVA) was performed to verify how the elementary school soccer class using VR technology affected students' attitude toward PE and flow in the class. Additionally, the results of the questionnaire were analyzed and the data that were deemed unreliable were excluded from analyses. Statistical significance was set at $p<0.05$. 


\section{Results}

5.1. The Effect of Experience of Elementary Soccer Class Using VR Technology on the Class Attitude

To investigate the effect of the VR sports room experience on class attitudes (interest/confidence/concentration) by sex (male/female) and group (VR sports room group/ traditional playground group), the mean and standard deviation of the sub-factors of the class attitude were analyzed with a three-way mixed ANOVA (Tables 6 and 7). Both groups showed a normal distribution.

Table 6. Mean and standard deviation for the class attitudes of each group.

\begin{tabular}{|c|c|c|c|c|c|c|c|c|}
\hline \multirow[b]{2}{*}{ Group } & \multirow[b]{2}{*}{ Sex } & \multicolumn{2}{|c|}{ Interest } & \multicolumn{2}{|c|}{ Confidence } & \multicolumn{2}{|c|}{ Concentration } & \multirow[b]{2}{*}{$\mathbf{n}$} \\
\hline & & $\begin{array}{c}\text { Pre- } \bar{X} \\
\text { (SD) }\end{array}$ & $\begin{array}{c}\text { Post- } \bar{X} \\
\text { (SD) }\end{array}$ & $\begin{array}{c}\text { Pre- } \bar{X} \\
\text { (SD) }\end{array}$ & $\begin{array}{c}\text { Post- } \bar{X} \\
\text { (SD) }\end{array}$ & $\begin{array}{c}\text { Pre- } \bar{X} \\
\text { (SD) }\end{array}$ & $\begin{array}{l}\text { Post- } \bar{X} \\
\text { (SD) }\end{array}$ & \\
\hline \multirow{3}{*}{$\begin{array}{l}\text { Experimental } \\
\text { group }\end{array}$} & Male & $\begin{array}{c}4.48 \\
(0.72)\end{array}$ & $\begin{array}{c}4.55 \\
(0.68)\end{array}$ & $\begin{array}{c}3.47 \\
(0.75)\end{array}$ & $\begin{array}{c}3.80 \\
(0.72)\end{array}$ & $\begin{array}{c}3.28 \\
(0.79)\end{array}$ & $\begin{array}{c}3.64 \\
(0.78)\end{array}$ & 25 \\
\hline & Female & $\begin{array}{c}4.22 \\
(0.81)\end{array}$ & $\begin{array}{c}4.24 \\
(0.99) \\
\end{array}$ & $\begin{array}{c}3.23 \\
(0.96) \\
\end{array}$ & $\begin{array}{c}3.47 \\
(0.91) \\
\end{array}$ & $\begin{array}{c}3.58 \\
(0.78) \\
\end{array}$ & $\begin{array}{c}3.89 \\
(0.80) \\
\end{array}$ & 25 \\
\hline & Total & $\begin{array}{c}4.35 \\
(0.77) \\
\end{array}$ & $\begin{array}{c}4.39 \\
(0.86) \\
\end{array}$ & $\begin{array}{c}3.35 \\
(0.86) \\
\end{array}$ & $\begin{array}{c}3.63 \\
(0.83) \\
\end{array}$ & $\begin{array}{c}3.43 \\
(0.79) \\
\end{array}$ & $\begin{array}{c}3.76 \\
(0.79) \\
\end{array}$ & 50 \\
\hline \multirow{3}{*}{$\begin{array}{l}\text { Control } \\
\text { group }\end{array}$} & Male & $\begin{array}{c}4.64 \\
(0.68)\end{array}$ & $\begin{array}{c}4.69 \\
(0.65)\end{array}$ & $\begin{array}{c}4.02 \\
(0.90)\end{array}$ & $\begin{array}{c}4.00 \\
(0.80)\end{array}$ & $\begin{array}{c}4.19 \\
(0.76)\end{array}$ & $\begin{array}{c}4.23 \\
(0.73)\end{array}$ & 34 \\
\hline & Female & $\begin{array}{c}4.22 \\
(0.74)\end{array}$ & $\begin{array}{c}4.16 \\
(0.89)\end{array}$ & $\begin{array}{c}3.42 \\
(0.80)\end{array}$ & $\begin{array}{l}3.45 \\
(0.95)\end{array}$ & $\begin{array}{c}3.91 \\
(0.71)\end{array}$ & $\begin{array}{c}3.96 \\
(0.74)\end{array}$ & 29 \\
\hline & Total & $\begin{array}{c}4.45 \\
(0.73) \\
\end{array}$ & $\begin{array}{c}4.45 \\
(0.81) \\
\end{array}$ & $\begin{array}{l}3.75 \\
(0.90)\end{array}$ & $\begin{array}{c}3.74 \\
(0.91) \\
\end{array}$ & $\begin{array}{c}4.06 \\
(0.75) \\
\end{array}$ & $\begin{array}{c}4.11 \\
(0.74) \\
\end{array}$ & 63 \\
\hline \multirow{3}{*}{ Total } & Male & $\begin{array}{c}4.57 \\
(0.70)\end{array}$ & $\begin{array}{c}4.63 \\
(0.66)\end{array}$ & $\begin{array}{c}3.79 \\
(0.88) \\
\end{array}$ & $\begin{array}{c}3.91 \\
(0.77)\end{array}$ & $\begin{array}{c}3.80 \\
(0.89) \\
\end{array}$ & $\begin{array}{c}3.98 \\
(0.80) \\
\end{array}$ & 59 \\
\hline & Female & $\begin{array}{c}4.22 \\
(0.76)\end{array}$ & $\begin{array}{c}4.20 \\
(0.93)\end{array}$ & $\begin{array}{c}3.33 \\
(0.88)\end{array}$ & $\begin{array}{c}3.46 \\
(0.92)\end{array}$ & $\begin{array}{c}3.76 \\
(0.75) \\
\end{array}$ & $\begin{array}{c}3.93 \\
(0.76) \\
\end{array}$ & 54 \\
\hline & Total & $\begin{array}{c}4.40 \\
(0.75)\end{array}$ & $\begin{array}{c}4.42 \\
(0.83)\end{array}$ & $\begin{array}{c}3.57 \\
(0.90)\end{array}$ & $\begin{array}{c}3.69 \\
(0.87)\end{array}$ & $\begin{array}{c}3.76 \\
(0.83)\end{array}$ & $\begin{array}{c}3.95 \\
(0.78)\end{array}$ & 113 \\
\hline
\end{tabular}

Table 7. Results of the ANOVA concerning students' attitude.

\begin{tabular}{|c|c|c|c|c|}
\hline & & Interest & Confidence & Concentration \\
\hline \multirow[t]{4}{*}{$\begin{array}{c}\text { Interaction } \\
\text { effect }\end{array}$} & $\begin{array}{c}\text { Time } \times \text { Group } \times \\
\text { Sex }\end{array}$ & $\begin{array}{c}F(1,109)=0.065 \\
p=0.800\end{array}$ & $\begin{array}{c}F(1,109)=0.283 \\
p=0.596\end{array}$ & $\begin{array}{c}F(1,109)=0.048 \\
p=0.827\end{array}$ \\
\hline & Time $\times$ Group & $\begin{array}{c}F(1,109)=0.170 \\
p=0.681\end{array}$ & $\begin{array}{c}F(1,109)=4.369 \\
\quad p=0.039 *\end{array}$ & $\begin{array}{c}F(1,109)=4.479 \\
\quad p=0.037^{*}\end{array}$ \\
\hline & Group $\times$ Sex & $\begin{array}{c}F(1,109)=0.475 \\
p=0.492\end{array}$ & $\begin{array}{c}F(1,109)=0.957 \\
p=0.330\end{array}$ & $\begin{array}{c}F(1,109)=4.692 \\
p=0.032 *\end{array}$ \\
\hline & Time $\times$ Group & $\begin{array}{c}F(1,109)=0.495 \\
p=0.483\end{array}$ & $\begin{array}{c}F(1,109)=0.013 \\
p=0.910\end{array}$ & $\begin{array}{c}F(1,109)=0.029 \\
p=0.866\end{array}$ \\
\hline \multirow[t]{3}{*}{ Main effect } & Time & $\begin{array}{c}F(1,109)=0.113 \\
p=0.737\end{array}$ & $\begin{array}{c}F(1,109)=4.313 \\
p=0.040 *\end{array}$ & $\begin{array}{c}F(1,109)=7.488 \\
p=0.007^{* *}\end{array}$ \\
\hline & Group & $\begin{array}{c}F(1,109)=0.165 \\
p=0.685\end{array}$ & $\begin{array}{c}F(1,109)=2.504 \\
p=0.116\end{array}$ & $\begin{array}{c}F(1,109)=14.080 \\
p<0.001\end{array}$ \\
\hline & Sex & $\begin{array}{c}F(1,109)=7.746 \\
p=0.006^{* *}\end{array}$ & $\begin{array}{c}F(1,109)=8.399 \\
p=0.005^{* *}\end{array}$ & $\begin{array}{c}F(1,109)=0.001 \\
p=0.979\end{array}$ \\
\hline
\end{tabular}




\subsubsection{Effects of VR Technology on Students' Interest}

Table 7 shows that only the main effect of Sex was significant.

\subsubsection{Effects of VR Technology on Students' Confidence}

For confidence, there was not a statistically significant three-way interaction; however, we continued the analysis because there was a significant time by group interaction (Table 7 , Figure 2). In other words, the group participating in the VR sports room class displayed significantly increased confidence than the group participating in the playground class. The main effects according to time and sex were also significant.

\section{Confidence}

4.0

3.8

3.6

3.4

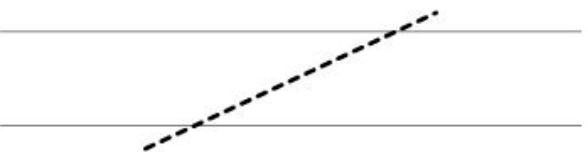

3.2

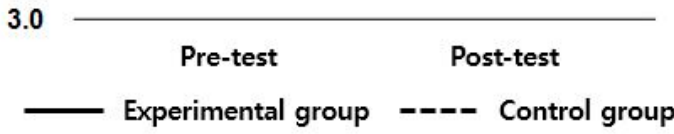

Figure 2. Mean plots for the confidence test.

\subsubsection{Effects of VR Technology on Students' Concentration}

For concentration, there was not a statistically significant three-way interaction; however, we continued the analysis because there were significant time by group and group by sex interactions (Table 7; Figures 3 and 4). These results imply that the group that participated in the VR sports class concentrated more than the participants in the playground class. In addition, the female students showed higher concentration in the experimental group, and the male students showed higher concentration in the control group. Main effects according to time $(\mathrm{F}(1109)=7.488, p=0.007)$ and $\operatorname{Group}(\mathrm{F}(1109)=14.080, p<0.001)$ were significant.

\section{Concentration}

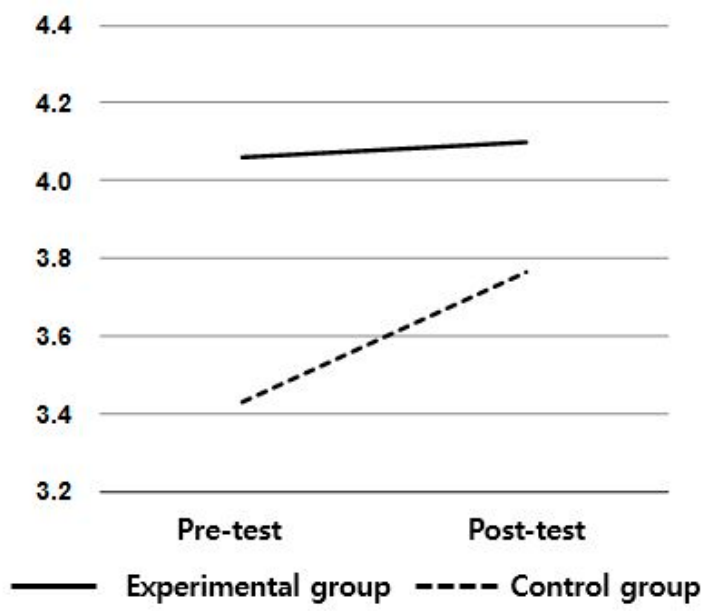

Figure 3. Mean plots for the concentration test per Time and Group. 


\section{Concentration}

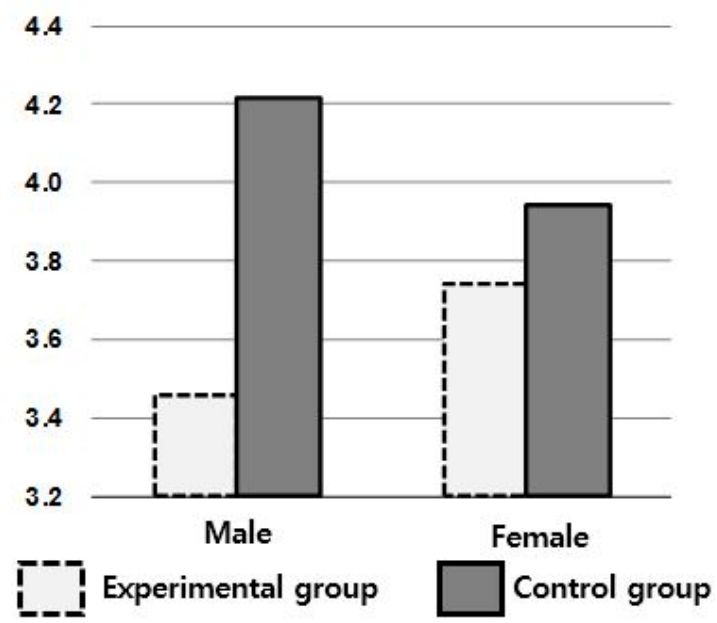

Figure 4. Mean Plots for the concentration test per Sex and Group.

\subsection{The Effect of VR Technology on Students' Flow in Class}

To investigate the effect of VR sports room experience on students' flow in class (self-directed experience/attention to PE class/integration of PE classes/consensus of class behavior and conscious/challenge and sense of control) by sex (male/female) and group (VR sports room group/traditional playground group), the mean and standard deviation of the sub-factors of the flow in class were analyzed with a three-way mixed ANOVA (Tables 8 and 9). Both groups showed a normal distribution.

Table 8. Mean and standard deviation for the flow in class of each group.

\begin{tabular}{|c|c|c|c|c|c|c|c|}
\hline \multirow[b]{2}{*}{ Sub-Factor } & \multirow{2}{*}{$M(S D)$} & \multicolumn{3}{|c|}{ Experimental Group } & \multicolumn{3}{|c|}{ Control Group } \\
\hline & & $\begin{array}{c}\text { Male } \\
(n=25)\end{array}$ & $\begin{array}{l}\text { Female } \\
(n=25)\end{array}$ & $\begin{array}{c}\text { Total } \\
(N=50)\end{array}$ & $\begin{array}{c}\text { Male } \\
(n=34)\end{array}$ & $\begin{array}{l}\text { Female } \\
(n=29)\end{array}$ & $\begin{array}{c}\text { Total } \\
(\mathrm{N}=63)\end{array}$ \\
\hline \multirow{2}{*}{ Self-directed experience } & $\begin{array}{c}\text { Pre- } \bar{X} \\
\text { (SD) }\end{array}$ & $\begin{array}{c}4.40 \\
(0.76)\end{array}$ & $\begin{array}{c}4.30 \\
(0.79)\end{array}$ & $\begin{array}{c}4.35 \\
(0.77)\end{array}$ & $\begin{array}{c}4.58 \\
(0.63)\end{array}$ & $\begin{array}{c}4.12 \\
(0.70)\end{array}$ & $\begin{array}{c}4.37 \\
(0.70)\end{array}$ \\
\hline & $\begin{array}{l}\text { Post- } \bar{X} \\
\text { (SD) }\end{array}$ & $\begin{array}{c}4.54 \\
(0.58)\end{array}$ & $\begin{array}{c}4.41 \\
(0.72)\end{array}$ & $\begin{array}{c}4.47 \\
(0.65)\end{array}$ & $\begin{array}{c}4.75 \\
(0.62)\end{array}$ & $\begin{array}{c}4.25 \\
(0.83)\end{array}$ & $\begin{array}{c}4.52 \\
(0.76)\end{array}$ \\
\hline \multirow{2}{*}{$\begin{array}{l}\text { Attention in physical } \\
\text { education class }\end{array}$} & $\begin{array}{c}\text { Pre- } \bar{X} \\
(\mathrm{SD})\end{array}$ & $\begin{array}{c}3.52 \\
(0.76)\end{array}$ & $\begin{array}{c}3.63 \\
(0.58)\end{array}$ & $\begin{array}{c}3.58 \\
(0.67)\end{array}$ & $\begin{array}{c}4.29 \\
(0.73)\end{array}$ & $\begin{array}{c}3.68 \\
(0.68)\end{array}$ & $\begin{array}{c}4.01 \\
(0.76)\end{array}$ \\
\hline & $\begin{array}{l}\text { Post- } \bar{X} \\
\text { (SD) }\end{array}$ & $\begin{array}{c}4.06 \\
(0.75) \\
\end{array}$ & $\begin{array}{c}4.01 \\
(0.69) \\
\end{array}$ & $\begin{array}{c}4.04 \\
(0.71)\end{array}$ & $\begin{array}{c}4.34 \\
(0.75)\end{array}$ & $\begin{array}{c}3.88 \\
(0.69) \\
\end{array}$ & $\begin{array}{c}4.13 \\
(0.75)\end{array}$ \\
\hline \multirow{2}{*}{$\begin{array}{l}\text { Integration of physical } \\
\text { education classes }\end{array}$} & $\begin{array}{l}\text { Pre- } \bar{X} \\
(\mathrm{SD})\end{array}$ & $\begin{array}{c}3.63 \\
(0.74) \\
\end{array}$ & $\begin{array}{c}3.62 \\
(0.72) \\
\end{array}$ & $\begin{array}{c}3.62 \\
(0.72) \\
\end{array}$ & $\begin{array}{c}4.17 \\
(0.91) \\
\end{array}$ & $\begin{array}{c}3.71 \\
(0.72) \\
\end{array}$ & $\begin{array}{c}3.96 \\
(0.85) \\
\end{array}$ \\
\hline & $\begin{array}{l}\text { Post- } \bar{X} \\
\text { (SD) }\end{array}$ & $\begin{array}{c}4.07 \\
(0.73)\end{array}$ & $\begin{array}{c}3.95 \\
(0.79)\end{array}$ & $\begin{array}{c}4.01 \\
(0.75)\end{array}$ & $\begin{array}{c}4.30 \\
(0.75)\end{array}$ & $\begin{array}{c}3.74 \\
(0.85)\end{array}$ & $\begin{array}{c}4.04 \\
(0.84)\end{array}$ \\
\hline \multirow{2}{*}{$\begin{array}{l}\text { Consensus of class } \\
\text { behavior and conscious }\end{array}$} & $\begin{array}{l}\text { Pre- } \bar{X} \\
(\mathrm{SD})\end{array}$ & $\begin{array}{c}3.77 \\
(0.77)\end{array}$ & $\begin{array}{l}3.59 \\
(0.78)\end{array}$ & $\begin{array}{l}3.68 \\
(0.77)\end{array}$ & $\begin{array}{c}4.17 \\
(0.79)\end{array}$ & $\begin{array}{c}3.54 \\
(0.69)\end{array}$ & $\begin{array}{c}3.88 \\
(0.81)\end{array}$ \\
\hline & $\begin{array}{l}\text { Post- } \bar{X} \\
\text { (SD) }\end{array}$ & $\begin{array}{c}3.92 \\
(0.79)\end{array}$ & $\begin{array}{c}3.76 \\
(0.87)\end{array}$ & $\begin{array}{c}3.84 \\
(0.82)\end{array}$ & $\begin{array}{c}4.28 \\
(0.75)\end{array}$ & $\begin{array}{c}3.72 \\
(0.77)\end{array}$ & $\begin{array}{c}4.02 \\
(0.80)\end{array}$ \\
\hline \multirow{2}{*}{$\begin{array}{l}\text { Challenge and sense of } \\
\text { control }\end{array}$} & $\begin{array}{l}\text { Pre- } \bar{X} \\
(\mathrm{SD})\end{array}$ & $\begin{array}{l}3.63 \\
(0.75)\end{array}$ & $\begin{array}{l}3.67 \\
(0.71)\end{array}$ & $\begin{array}{l}3.65 \\
(0.72)\end{array}$ & $\begin{array}{c}4.11 \\
(0.81)\end{array}$ & $\begin{array}{l}3.65 \\
(0.76)\end{array}$ & $\begin{array}{l}3.90 \\
(0.81)\end{array}$ \\
\hline & $\begin{array}{c}\text { Post- } \bar{X} \\
\text { (SD) }\end{array}$ & $\begin{array}{c}3.99 \\
(0.77)\end{array}$ & $\begin{array}{c}4.09 \\
(0.73)\end{array}$ & $\begin{array}{c}4.04 \\
(0.75)\end{array}$ & $\begin{array}{c}4.21 \\
(0.77)\end{array}$ & $\begin{array}{c}3.68 \\
(0.82)\end{array}$ & $\begin{array}{c}3.96 \\
(0.83)\end{array}$ \\
\hline
\end{tabular}


Table 9. Results of the ANOVA concerning students' flow in class.

\begin{tabular}{|c|c|c|c|c|}
\hline & & $\begin{array}{l}\text { Self-Directed } \\
\text { Experience }\end{array}$ & $\begin{array}{l}\text { Attention in Physical } \\
\text { Education Class }\end{array}$ & $\begin{array}{l}\text { Integration of Physical } \\
\text { Education Classes }\end{array}$ \\
\hline \multirow{4}{*}{ Interaction effect } & Time $\times$ Group $\times$ Sex & $\begin{array}{c}F(1,109)=0.005 \\
p=0.943\end{array}$ & $\begin{array}{c}F(1,109)=1.118 \\
\quad p=0.293\end{array}$ & $\begin{array}{c}F(1,109)=0.003 \\
p=0.953\end{array}$ \\
\hline & Time $\times$ Group & $\begin{array}{c}F(1,109)=0.055 \\
\quad p=0.815\end{array}$ & $\begin{array}{c}F(1,109)=5.444 \\
\quad p=0.021 *\end{array}$ & $\begin{array}{c}F(1,109)=4.326 \\
\quad p=0.040 *\end{array}$ \\
\hline & Group $\times$ Sex & $\begin{array}{c}F(1,109)=2.224 \\
p=0.139\end{array}$ & $\begin{array}{c}F(1,109)=5.923 \\
\quad p=0.017^{*}\end{array}$ & $\begin{array}{c}F(1,109)=2.965 \\
\quad p=0.088\end{array}$ \\
\hline & Time $\times$ Group & $\begin{array}{c}F(1,109)=0.105 \\
p=0.746\end{array}$ & $\begin{array}{c}F(1,109)=0.000 \\
\quad p=0.999\end{array}$ & $\begin{array}{c}F(1,109)=0.508 \\
\quad p=0.478\end{array}$ \\
\hline \multirow{3}{*}{ Main effect } & Time & $\begin{array}{c}F(1,109)=5.468 \\
\quad p=0.021 *\end{array}$ & $\begin{array}{c}F(1,109)=16.373 \\
\quad p<0.001^{* * *}\end{array}$ & $\begin{array}{c}F(1,109)=10.294 \\
\quad p=0.002^{* *}\end{array}$ \\
\hline & Group & $\begin{array}{c}F(1,109)=0.025 \\
\quad p=0.875\end{array}$ & $\begin{array}{c}F(1,109)=4.550 \\
\quad p=0.035 *\end{array}$ & $\begin{array}{c}F(1,109)=1.585 \\
\quad p=0.211\end{array}$ \\
\hline & Sex & $\begin{array}{c}F(1,109)=5.973 \\
p=0.016^{*}\end{array}$ & $\begin{array}{c}F(1,109)=4.790 \\
p=0.031^{*}\end{array}$ & $\begin{array}{c}F(1,109)=4.893 \\
p=0.029^{*}\end{array}$ \\
\hline
\end{tabular}

\subsubsection{Effects of VR Technology on Students' Self-Directed Experience}

For self-directed experience, no significant interaction effects were found for Sex by Group (Table 9). However, the main effects of Time and Sex were significant.

\subsubsection{Effects of VR Technology on Students' Attention in PE Class}

For attention, there was not a statistically significant three-way interaction; however, we continued the analysis because there were significant interaction effects for time by group and group by sex (Table 9; Figures 5 and 6). In other words, the group who participated in the VR sports class were more attentive than the participants in the playground class. Moreover, in the experimental group, the female students displayed higher attention; while, in the control group, the male students displayed higher attention. The main effects according to time, group, and sex were significant.

\section{Attention in PE}

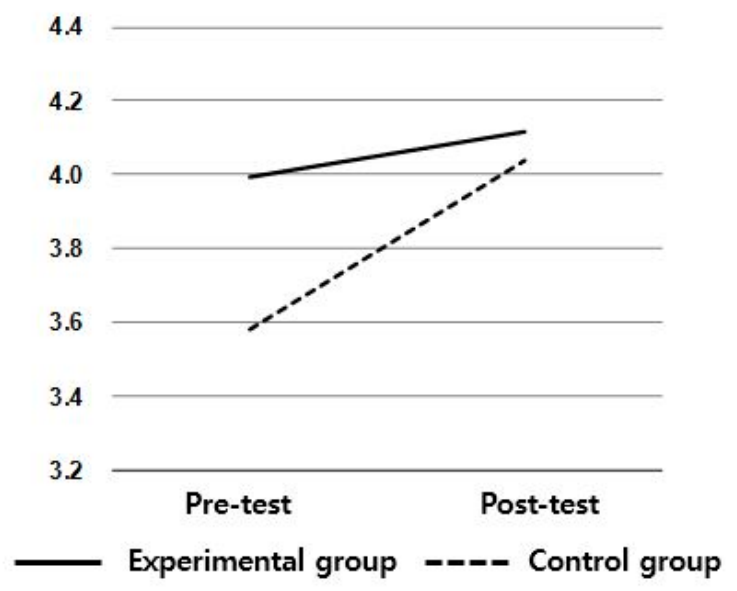

Figure 5. Mean plots for students' attention in the PE class per time and group. 


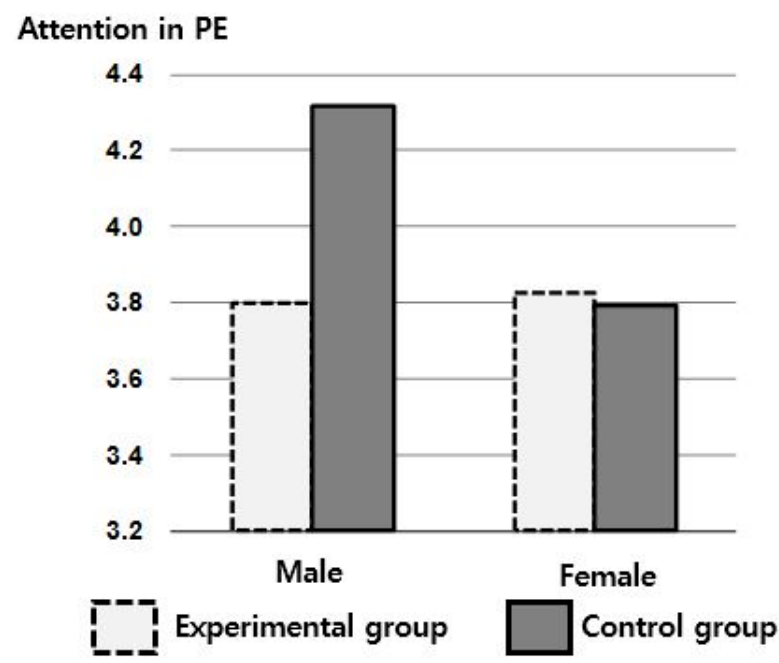

Figure 6. Mean plots for students' attention in the PE class per Sex and Group.

\subsubsection{Effects of VR Technology on Students' Integration in PE Classes}

For integration, there was not a statistically significant three-way interaction; however, we continued the analysis because there was a significant time by group interaction (Table 9; Figure 7). In other words, the group participating in the VR sports room class was more integrated with the PE classes than the group participating in the playground class. The main effects of the time and sex were significant.

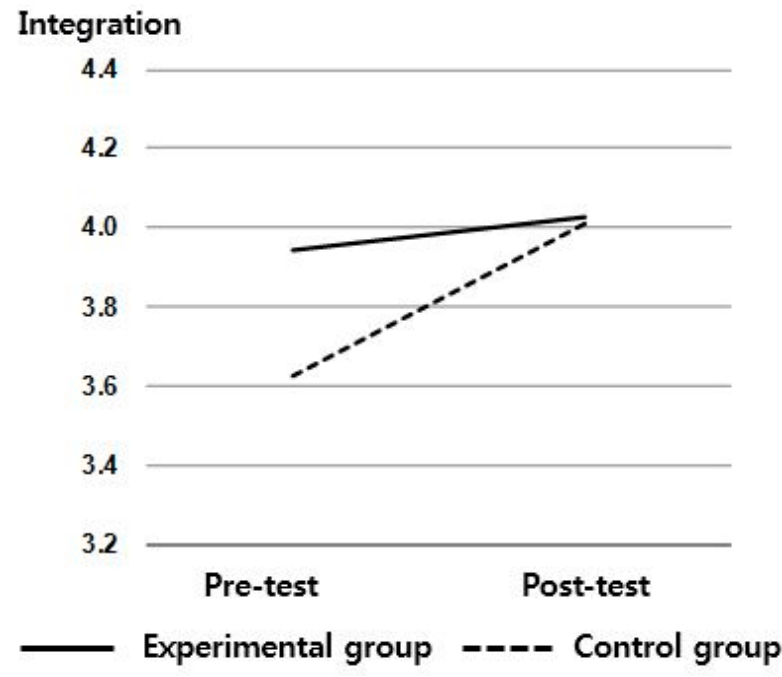

Figure 7. Mean plots for students' integration in PE classes.

5.2.4. Effects of VR Technology on Students' Consensus of Class Behavior and Consciousness

For consensus of class behavior and consciousness, no significant interaction was found for sex and group (Table 9); however, the main effects of time and sex were significant.

\subsubsection{Effects of VR Technology on Students' Challenge and Sense of Control}

For challenge and sense of control, there were significant time by group and group by sex, interactions (Table 9; Figures 8 and 9). In other words, the participants who participated in the VR sports room classes had a much higher level of challenge and sense of control than the participants in the playground class. The main effect of time was also significant. 


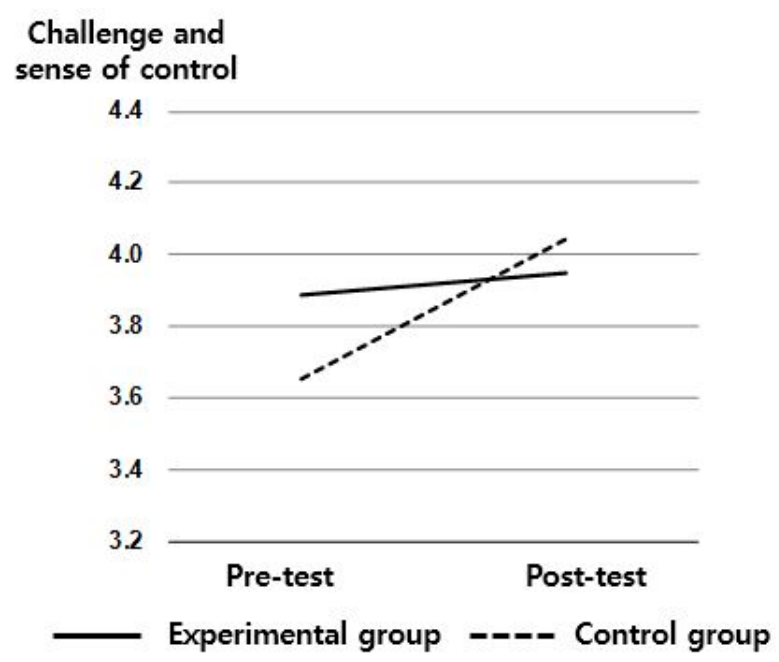

Figure 8. Mean plots for challenge and sense of control per time and group.

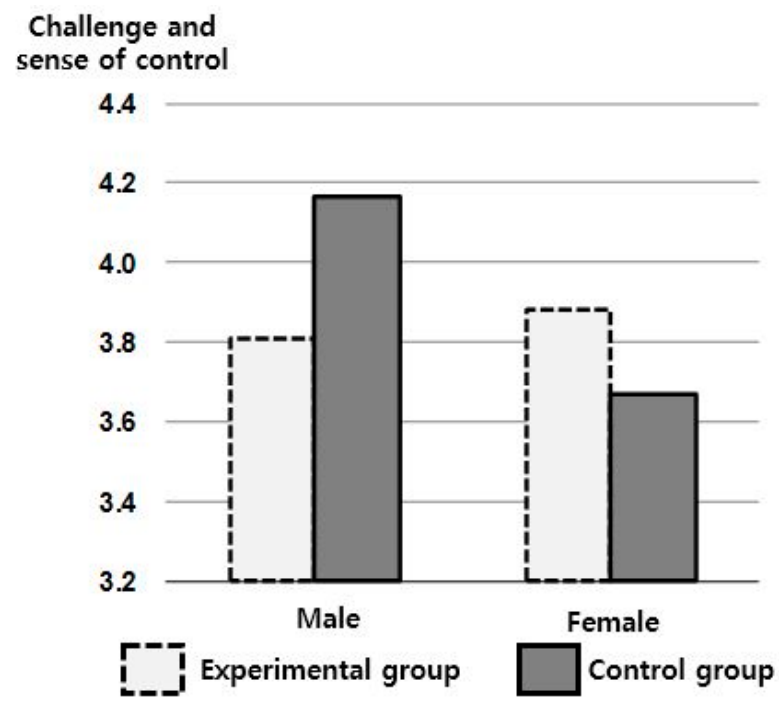

Figure 9. Mean plots for challenge and sense of control per Sex and Group.

\section{Discussion}

The purpose of this study was to verify the effect of elementary school soccer classes using VR technology on students' class attitude and flow in class. Significant results were shown concerning students' confidence and concentration, which were sub-factors of class attitude; as well as about attention, integration, challenge, and sense of control, which were sub-factors of flow in class. There were, however, no significant differences in students' interest-a sub-factor of class attitude-or self-directed experience and consensus of class behavior and conscious-sub-factors of flow in class.

\subsection{The Effect of VR Technology on Students' Attitude toward Their Soccer Class}

Students have fun using VR technology as an activity itself; however, afterward, they feel the pleasure and joy from learning [29]. Additionally, classes using VR technology are more suitable for constructing a self-directed learning environment because they are more realistic than other classes that use existing multimedia materials [30]. Thus, the use of VR technology seems to positively affect learners' interest.

However, in this study, contrary to our hypothesis, there was no significant difference between groups concerning interest.

Similar results were obtained in previous studies by Parong and Mayer [31] and Zhang and colleagues [32]. While surveys have shown a slight increase in students' interest, their 
overall interest levels remain low; Noh and colleagues [33] found that increasing students' interest in general learning is difficult to change over a brief period. In a study by Irene and colleagues [34], it was suggested that the students would be more interested in PE classes if they had enough time to learn sports and games. In this study, perhaps five classes were too few to effectively spark students' interest. Therefore, future studies should expand the length of the program to ensure students are more enthusiastic about the program.

The results showed that the group using VR technology displayed more confidence than did the traditional PE group. This is similar to the results of a previous study [35], in which students used VR technology to perform various tasks themselves, prevent risks and injuries associated with physical activities, and provide a safe learning environment. Additionally, the difficulty control function of VR technology was associated with increased confidence. In other words, students who have already succeeded through an adjustment of the challenge settings are gradually increasing their achievement level and self-evaluation. Therefore, since the soccer class using VR technology positively affected students' confidence in PE class, the change in attitude may have improved their motivation for physical activity and the opportunity for participation.

There was also a significant increase in students' concentration, another sub-factor of class attitude. In traditional PE classes, students typically follow the guidance and direction of the teacher. On the other hand, in classes using VR technology, the students can learn individually at varied times. Typically, lessons using VR technology are conducted according to individual learners or group learners, and the learners frequently ask questions to teachers [36]; therefore, it seems that learners' autonomy may be associated with their concentration.

\subsection{The Effect of VR Technology on Students' Flow in Soccer Class}

There were no significant group differences for "self-directed experience" and "consensus of class behavior and conscious", which are sub-factors of flow in class. Five 40-min lessons each week seemed to be a relatively short time to improve "self-directed experience" and "consensus of class behavior and conscious". Triguerosand colleagues [37] said that these two sub-factors of flow are the sub-factors with the highest intensity and the most time-consuming.

According to Cha and colleagues [38], at present, the VR sports room is less immersed in PE classes because of the limited number of sports rooms and software programs that can be used in classes. Therefore, in the PE class using VR technology, the technique that reproduces the virtual environment as reality is the most important factor to increase flow. Parong and Mayer [31] said that it is necessary to introduce programs that are simpler and more liberal to increase students' flow in the class using VR technology. Therefore, students might be more immersed if a VR program implements an actual terrain slope or develops a variety of programs that embody real-world environments, such as the characteristics of specific fields, arenas, or clubs that vary in seasons and weather.

Another reason for hindering strong commitment is waiting time. The VR sports room is limited in space compared to a wide playground. Because of these spatial constraints, students' waiting times become longer and students cannot immerse themselves in the class. Thus, soccer classes using VR technology should improve the quality of the programs and reduce spatial limitations to increase elementary students' attitudes toward PE classes as well as their flow in class.

Students exposed to the VR technology displayed higher attention than those who received traditional instruction. This means that PE classes using VR technology can materialize things that are impossible in reality; therefore, students can be more involved in learning than traditional PE classes led by teachers. These results are consistent with previous studies $[4,39]$ that revealed that learners' sensory involvement in VR is stronger than in general classes.

We also revealed a significant difference between the integration of students in both groups. The reason that the PE class using VR technology was more effective in integrating 
students than the traditional PE class was consistent with a previous study $[33,39]$ that showed that the VR learning process is more important than the result. Carruth [40] also mentioned that PE classes using VR technology reduce various anxieties about injuries, which may be obstacles to participation in PE activities. In addition, it can naturally induce the acquisition of exercise skills and promote integration because VR technology provides repetitive and direct feedback on a particular sport. Consequently, students are intrinsically motivated, which may promote continued learning in the future.

Finally, challenge and sense of control were significantly increased among those who received VR instruction. As mentioned above, students can improve their confidence in PE classes using VR technology because they can control the difficulty level. This difficulty control function has a positive effect on the exercise performance ability of students with low motor skills, and students receive positive feedback from other students [41]. Therefore, students who have increased their drive to be physically active become more interested in PE and will be more involved in physical activity [42]. This is similar to the results of a previous study [43], in which the higher the motor skill the higher the degree of fun in PE classes (and vice versa). Additionally, interaction with VR content using a real-world interface promotes active learning, thereby enhancing the applicability of knowledge [44]. Therefore, VR technology may challenge students to learn and their control over the activities fosters immersion and thus flow.

\section{Conclusions}

The purpose of this study was to investigate the effect of using VR technology in an elementary school soccer class on students' attitudes toward PE class and flow in class. We examined fifth-grade students from " $\mathrm{A}$ " elementary school, equipped with a VR sports room, and fifth-grade students from " $\mathrm{B}$ " elementary school, where traditional PE classes are conducted. Soccer was chosen as the sport because the program was well developed by the VR company, and elementary school students' participation in soccer was high.

First, there was no significant difference in students' interest between groups, possibly because we only conducted five classes; therefore, the hypothesis that the soccer class using VR technology would spark students' interest was rejected.

Second, students in the VR technology class (vs. traditional) showed higher confidence and concentration. With the difficulty control function, students who do not like to exercise, students with disabilities, or students with low motor skills can enjoy the same classes as students with excellent athletic ability. This, therefore, allows students to experience a variety of successes. Additionally, VR sports provide students with a safe learning environment that prevents the risks and injuries associated with physical activity; thus, it motivates students' participation in physical activity. Therefore, the hypothesis that soccer classes using VR technology would promote students' confidence and concentration was accepted.

Third, students in the VR technology class (vs. traditional) showed no significant difference in self-directed experience or consensus of class behavior and consciousness. This might be because of the lack of VR sports programs currently being developed, the simplicity of the program, or the short lesson duration. Further, the fact that many students waited in front of a single screen could be an obstacle to students' immersion. Therefore, the hypothesis that soccer classes using VR technology would affect the self-directed experience and consensus of class behavior and consciousness was rejected.

Fourth, students in the VR technology class (vs. traditional) showed a significant difference in attention. This means that students' PE classes using VR technology can be more focused on learning than traditional PE classes because they can materialize things that are impossible in reality. Therefore, the hypothesis that soccer classes using VR technology would affect students' attention in PE class was accepted.

Fifth, students in the VR technology class (vs. traditional) showed a significant difference in class integration. This is because VR technology provides repetitive and direct 
feedback on a particular sport. Therefore, the hypothesis that soccer classes using VR technology would promote students' integration was accepted.

Sixth, students in the VR technology class (vs. traditional) showed a significant difference in challenge and sense of control. VR sports provide a variety of successful experiences for students with low motor skills because they can control difficulty levels. Therefore, students received positive feedback from teachers, and their enjoyment in the class was increased, which would have a positive effect on the sense of challenge and control. Therefore, the hypothesis that soccer classes using VR technology would affect students' sense of challenge and control was accepted.

Based on the conclusions of this study, several implications for subsequent studies are suggested. First, this study was limited to elementary school students located in Seoul and Gyeonggi province; therefore, it is not possible to generalize the results to elementary school students across the country. In subsequent studies, students from various regions should be included. Second, we only examined soccer classes. In future research, it is necessary to conduct detailed research on VR use across various sports. Third, this study was conducted for a short period (five weeks) and in a few classes; therefore, it is necessary to examine VR and its effects over longer periods and more classes. Fourth, we failed to examine, in depth, students' opinions of the lessons or the truthfulness of their responses. Therefore, it is necessary to conduct qualitative research or mixed research simultaneously. Lastly, this study only measured attitudes and flow in PE, which are students' affective domains for PE. Therefore, further studies need to study how soccer classes using VR affect the improvement of students' soccer skills (individual or team skills).

Author Contributions: Conceptualization, H.S.L. and J.L.; methodology, H.S.L.; writing-original draft preparation, H.S.L. and J.L.; writing-review and editing, H.S.L. and J.L.; visualization, H.S.L.; supervision, H.S.L. and J.L. All authors have read and agreed to the published version of the manuscript.

Funding: This research received no external funding.

Institutional Review Board Statement: Not applicable.

Informed Consent Statement: Not applicable.

Data Availability Statement: All data used for the study were publicly available data retrieved by the author.

Conflicts of Interest: The authors declare no conflict of interest.

\section{References}

1. Gawrisch, D.P.; Richards, K.A.R.; Killian, C.M. Integrating Technology in Physical Education Teacher Education: A Socialization Perspective. QUEST 2020, 72, 260-277. [CrossRef]

2. Palao, J.M.; Hastie, P.A.; Cruz, P.G.; Ortega, E. The Impact of Video Technology on Student Performance in Physical Education. Technol. Pedagog. Educ. 2015, 24, 51-63. [CrossRef]

3. Opstoel, K.; Chapelle, L.; Prins, F.J.; Meester, A.D.; Haerens, L.; Tartwijk, J.V.; Martelaer, K.D. Personal and Social Development in Physical Edcuation and Sports: A Review Study. Eur. Phys. Educ. Rev. 2019, 26, 797-813. [CrossRef]

4. Lee, H.S.; Lee, J.H. The Effect of T-ball Class on Physical Self-Efficacy of Elementary School Students Using Virtual Reality Technology (VR). Korea J. Sports Sci. 2020, 29, 613-624. [CrossRef]

5. Renshaw, I.; Chow, J.Y. A Constraint-led Approach to Sport and Physical Education Pedagogy. Phys. Educ. Sport Pedagog. 2019, 24, 103-116. [CrossRef]

6. Wyant, J.; Beak, J.H. Re-thinking Technology Adoption in Physical Education. Curric. Stud. Health Phys. Educ. 2019, 10, 3-17. [CrossRef]

7. Trajkova, M.; Cafaro, F. Takes Tutu to ballet: Designing visual and verbal feedback for augmented mirrors. Proc. Acm Interact. Mob. Wearable Ubiquitous Technol. 2018, 2, 1-30. [CrossRef]

8. Chan, J.C.; Leung, H.; Tang, J.K.; Komura, T. A virtual reality dance training system using motion capture technology. IEEE Trans. Learn. Technol. 2010, 4, 187-195. [CrossRef]

9. Ochi, D.; Kameda, A.; Takahashi, K.; Makiguchi, M.; Takeuchi, K. VR technologies for rich sports experience. In ACM SIGGRAPH 2016 Emerging Technologies; Association for Computing Machinery: Anaheim, CA, USA, 2016; pp. 1-2. 
10. Gradl, S.; Eskofier, B.M.; Eskofier, D.; Mutshcler, C.; Otto, S. Virtual and augmented reality in sports: An overview and acceptance study, UbiComp '16. In Proceedings of the 2016 ACM International Joint Conference on Pervasive and Ubiquitous Computing; Association for Computing Machinery: Heidelberg, Germany, 2016; pp. 885-888. [CrossRef]

11. Park, S.W.; Kim, S.M.; Kim, Y.S. Current status and development plan of ICT convergence physical education class using virtual reality (VR) sports room. J. Learn. Cent. Curric. Instr. 2018, 18, 1003-1025. [CrossRef]

12. Chang, K.E.; Zhang, J.; Huang, Y.S.; Liu, T.C.; Sung, Y.T. Applying Augmented Reality in Physical Education on Motor Skills Learning. Interact. Learn. Environ. 2020, 28, 685-697. [CrossRef]

13. Tang, H.W.; Tsai, C.W. Emerging tools and applications of virtual reality in education. Int. J. E Adopt. 2016, 8, 56-60.

14. Cooper, G.; Park, H.; Nasr, Z.; Thong, L.P.; Johnson, R. Using Virtual Reality in the Classroom: Preservice Teachers' Perceptions of Its Use as a Teaching and Learning Tool. Educ. Media Int. 2019, 56, 1-13. [CrossRef]

15. Beck, D. Augmented and Virtual Reality in Education: Immersive Learning Research. J. Educ. Comput. Res. 2019, 57, 1619-1625. [CrossRef]

16. Edler, D.; Keil, J.; Wiedenlübbert, T.; Sossna, M.; Kühne, O.; Dickmann, F. Immersive VR Experience of Redeveloped PostIndustrial Sites: The Example of "Zeche Holland" in Bochum-Wattenscheid. J. Cartogr. Geogr. Inf. 2019, 69, 267-284. [CrossRef]

17. Hruby, F.; Castellanos, I.; Ressl, R. Cartographic Scale in Immersive Virtual Environments. J. Cartogr. Geogr. Inf. 2020. [CrossRef]

18. Siegle, D. Seeing is Believing: Using Virtual and Augmented Reality to Enhance Student Learning. Gift. Child Today 2019, 42, 46-52. [CrossRef]

19. Korean Ministry of Culture, Sports and Tourism. Spread Virtual Reality Sports Room in 178 Elementary Schools Nationwide. In Korean Ministry of Culture, Sports and Tourism; The Department of Sports Industry: Seoul, Korea, 2018.

20. Dube, B.A.; Nhamo, E.; Magonde, S. Factors affecting ICT integration in the Teaching and Learning of Physical Education in South Africa: A Case of Johannesburg East Cluster Primary Schools in the Gauteng Province. Int. J. SportExerc. Health Res. 2018, 2, 88-92. Available online: http://www.sportscienceresearch.com/IJSEHR_201821_03.pdf (accessed on 8 December 2020). [CrossRef]

21. Hannah, B.; Victoria, A. Barriers and facilitators to using digital technologies in the Cooperative Learning model in physical education. Phys. Educ. Sport Pedagog. 2017, 22, 563-579. [CrossRef]

22. Koekoek, J.; Mars, H.; Kamp, J.; Walinga, W.; Hilvoorde, I. Aligning Digital Video Technology with Game Pedagogy in Physical Education. J. Phys. Educ. Recreat. Danc. 2018, 89, 12-22. [CrossRef]

23. Adam, T.; Tatnall, A. The value of using ICT in the education of school students with learning difficulties. Educ. Inf. Technol. 2017, 22, 2711-2726. [CrossRef]

24. Korean Ministry of Culture, Sports and Tourism, Results of 2017 Survey on Participation in National Sports for All, Korea. Ministry of Culture, Sports and Tourism. 2017. Available online: https:/ /www.mcst.go.kr/kor/s_policy/dept/deptView.jsp? pSeq=1688\&pDataCD=0406000000 (accessed on 31 January 2018).

25. Jang, S.T. The Effects of Secondary Physical Education Personalized System for Instruction. Unpublished Master's Thesis, Graduate School of Education, Korea National University of Education, Seoul, Korea, 2006.

26. Hyun, S. The Effects of Image of Female Physical Education Teacher on Female Students' Physical Competence and Physical Education Class Attitude. Unpublished Master's Thesis, The Graduate School of Education, Ewha Womans University, Seoul, Korea, 2018.

27. Csikszentmihalyi, M. Beyond boredom and anxiety. Jossey-Bass; 25th Anniversary edition (15 April 2000).

28. Kim, J.H. Development and Validation of Flow Scale for Physical Education of Middle School Students. Korean Assoc. Sports Pedagog. 2008, 15, 65-81.

29. Kim, Y.R.; Choi, M.Y. The Effect of VR Contents on Elementary School Music Lesson. J. Music Educ. Sci. 2018, 35, 1-20. [CrossRef]

30. Shim, K.C.; Park, J.S.; Kim, H.S.; Kim, J.H.; Park, Y.C.; Ryu, H.I. Exploring Application Ways of Virtual Reality Technology in Science Education. J. Korean Assoc. Sci. Educ. 2001, 21, 725-737.

31. Parong, J.; Mayer, R.E. Learning science in immersive virtual reality. J. Educ. Psychol. 2018, 110, 785-797. [CrossRef]

32. Zhang, K.; Suo, J.; Chen, J.; Liu, X.; Gao, L. Design and implementation of fire safety education system on campus based on virtual reality technology. In Federated Conference on Computer Science and Information Systems (FedCSIS); IEEE: Prague, Czech Republic, 2017; pp. 1297-1300. [CrossRef]

33. Noh, K.H.; Jee, H.K.; Lim, S.H. Effect of Augmented Reality Contents Based Instruction on Academic Achievement, Interest and Flow of Learning. Korean Contents Soc. 2010, 10, 1-13. [CrossRef]

34. Irene, R.; Sixto, G.-V.; Javier, F.-R.; Natalia, M. Physical activity levels, game performance and friendship goals using two different pedagogical models: Sport Education and Direct Instruction. Phys. Educ. Sport Pedagog. 2019, 24, 87-102. [CrossRef]

35. Kang, S.; Kang, S. The study on the application of virtual reality in adapted physical education. Clust. Comput. 2019, 22, 2351-2355. [CrossRef]

36. Lee, J.M.; Kim, J.H. Development and Application of ICT Teaching Learning Material for Physical Education Applied to the Inquiry Learning Model. J. Korean Assoc. Inf. Educ. 2009, 13, 1-8.

37. Trigueros, R.; Aguilar-Parra, J.M.; Cangas, A.J.; López-Liria, R.; Álvarez, J.F. Influence of Physical Education Teachers on Motivation, Embarrassment and the Intention of Being Physically Active During Adolescence. Int. J. Environ. Res. Public Health 2019, 16, 2295. [CrossRef] [PubMed] 
38. Cha, H.J.; Kye, B.K.; Park, T.J. Analysis of facilitating and conflicting factors for the educational use of new ICT technologies. J. HCI Soc. Korea 2017, 21, 567-578.

39. Kim, D.J.; Jeon, S.J. Design and Application of a Virtual Reality based Teaching Model for Field Based Learning Activities. J. Korean Assoc. Inf. Educ. 2013, 18, 133-142.

40. Carruth, D.-W. Virtual reality for education and workforce training. In Proceedings of the 201715 th International Conference on Emerging eLearning Technologies and Applications (ICETA), Stary Smokovec, Slovakia, 26-27 October 2017; pp. 1-6. [CrossRef]

41. Lee, H.-S. The Effect of T-Ball Class Using Virtual Reality Sports Room on Pleasure in Physical Activity of Elementary School Students. Asian J. Phys. Educ. Sport Sci. 2020, 8, 39-53. Available online: https://www.kci.go.kr/kciportal/ci/sereArticleSearch/ ciSereArtiView.kci?sereArticleSearchBean.artiId=ART002645619 (accessed on 10 December 2020).

42. Kim, I.S. The Influence Competence Beliefs, Perception of Sport Ability, and Sport Value on Achievement Goal Orientations, and Intention of Physical Education Classes Participation in Elementary School Children. Korean J. Elem. Phys. Educ. 2013, 19, 79-93.

43. Park, J.G.; Choi, S.S.; Mun, C.I.; Moon, I.S. Sources of Enjoyment and Worry in Physical Education Class of Elementary School. Korean Soc. Sport Psychol. 2006, 17, 85-104.

44. Billinghurst, M. Shared Space: Explorations in Collaborative Augmented Reality. Unpublished Doctoral's Dissertation, University of Washington, Washington, DC, USA, 2002. 